\section{Síndrome de ALCAPA en adulto. Caso clínico}

\author{
HÉCTOR UGALDE P., SEBASTIÁN ROZAS A. ${ }^{a}$, \\ MARÍA IGNACIA SANHUEZA F.a, MARÍA CECILIA YUBINI L. ${ }^{a}$, \\ SEBASTIÁN GARCÍA B.
}

\section{Anomalous left coronary artery origin from the pulmonary artery causing angina. Report of one case}

Anomalies of the origin of coronary arteries are detected in $0.5-1.5 \%$ of all angiographies. Anomalous origin of the left main trunk is the most uncommon and its origin from pulmonary artery in adults is exceptional, usually because it is associated with a short survival. We report a 49-year-old female, presenting with a two months history of angina. The exercise electrocardiogram suggested ischemia. A coronary angiography was performed, showing the absence of the left main trunk in the left coronary sinus, a dilated right coronary artery, with no lesions and extensive collateral circulation to the anterior descending and circumflex arteries, with inverted flow and the left main trunk draining to the pulmonary artery. The left ventricle was mildly dilated with middle and apical anterior hypokinesia. Global systolic function was conserved. A surgical correction was decided, occluding the left main anomalous origin and performing a coronary artery bypass grafting from the left internal thoracic artery. The patient was discharged with no complications. At two years of follow-up she is symptom free and has a normal physical capacity.

(Rev Med Chile 2017; 145: 121-125)

Key words: Bland White Garland Syndrome; Cardiovascular Abnormalities; Coronary Vessel Anomalies.
Departamento Cardiovascular Hospital Clínico Universidad de Chile. Santiago, Chile. aEstudiante de Medicina. Escuela de Medicina. Universidad de Chile. Santiago, Chile.

Recibido el 2 de mayo de 2016 aceptado el 8 de septiembre de 2016.

Correspondencia a: Héctor Ugalde Prieto Departamento de Cardiología Hospital Clínico Universidad de Chile. Av. Santos Dumont 999. Santiago, Chile.
$\mathrm{E}$ 1 nacimiento anómalo de la arteria coronaria izquierda desde la arteria pulmonar (ALCAPA) es una alteración congénita muy infrecuente, cuya incidencia estimada es de 1 por cada 300.000 nacidos vivos ${ }^{1}$. Sin tratamiento, se describe una sobrevida en el primer año de vida de 10 a 15\%. Lo anterior, debido a la severa isquemia miocárdica que produce ${ }^{2}$. Esto hace extremadamente raro su hallazgo en adultos. Se han reportado sólo 151 casos hasta el año 2011, según una revisión del tema por Yau et al, lo que ha dificultado el estudio de esta condición en adultos ${ }^{3}$. En nuestro país, no hay casos descritos en pacientes adultos, lo que motiva esta presentación.

\section{Caso clínico}

Mujer de 49 años de edad, con antecedentes de tabaquismo activo, obesidad y dislipidemia en tratamiento. Consultó por un cuadro de dos meses de evolución de dolor retroesternal opresivo irradiado a mandíbula y epigastrio, de inicio en ejercicio de baja intensidad. El estaba asociado a síntomas neurovegetativos que cedían con el reposo. El examen físico era normal.

Se solicitaron exámenes generales (hemograma, perfil bioquímico, perfil lipídico, TSH), todos normales. Electrocardiograma también normal y radiografía de tórax anteroposterior que 


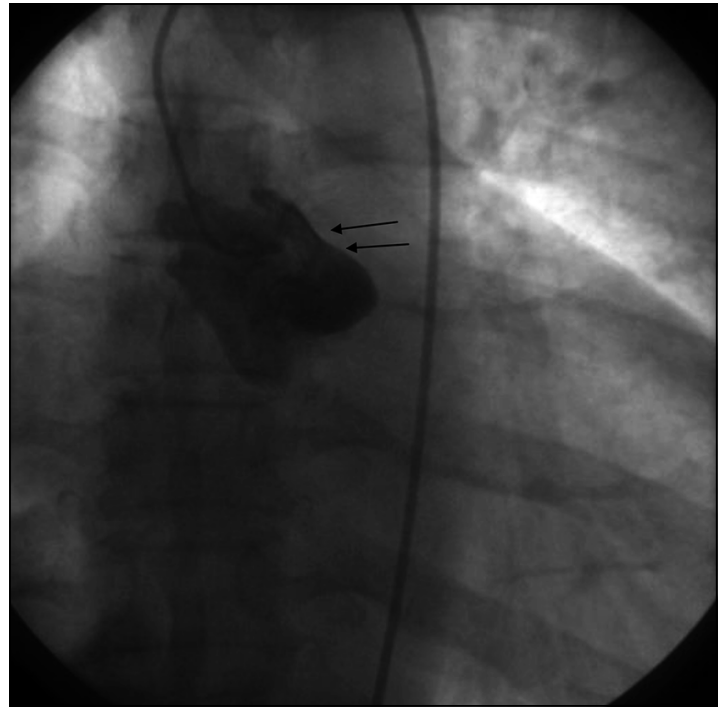

Figura 1. Ausencia $\mathrm{TCl}$ en seno coronariano izquierdo $(\longleftarrow)$.

evidenciaba una silueta cardíaca discretamente aumentada de tamaño a expensas del ventrículo izquierdo. Luego de estos resultados se continuó el estudio con un ecocardiograma transtorácico que mostró un ventrículo izquierdo levemente hipertrófico con función global conservada, fracción de eyección de 54,3\%. Se practicó test de esfuerzo, que fue detenido a los 5 minutos por dolor e infradesnivel significativo del segmento ST con frecuencia submáxima.

Dado los hallazgos, se solicitó estudio angiográfico coronario, el cual evidenció: ausencia del tronco coronario izquierdo (TCI) en el seno coronariano izquierdo (Figura 1) y una gran arteria coronaria derecha (ACD) hiperdominante, sin lesiones en su trayecto (Figura 2), que nutría y llenaba por completo la arteria coronaria izquierda (ACI) en forma retrógrada mediante un amplio sistema de circulación colateral, observándose además que el origen de la ACI (TCI) drenaba a nivel del tronco de la arteria pulmonar (AP) (Figura 3). Lo anterior concordante con el diagnóstico de síndrome de ALCAPA.

Con el diagnóstico de ALCAPA confirmado, la paciente fue sometida a cirugía de revascularización miocárdica con implante de un bypass desde la arteria mamaria interna izquierda hacia la porción proximal de la arteria descendente an-

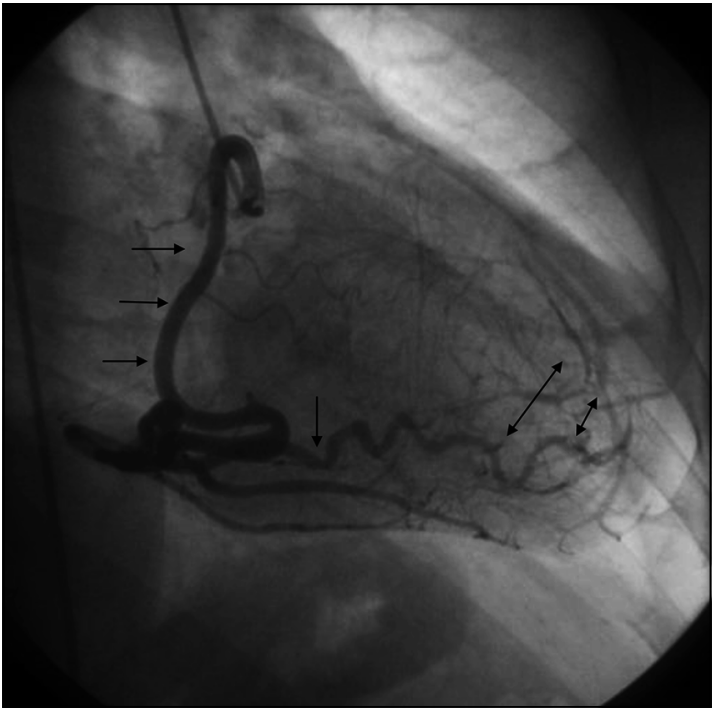

Figura 2. Arteria coronaria derecha de importante desarrollo $(\longrightarrow)$ con circulación colateral a $\mathrm{ACl}(\longleftrightarrow)$.

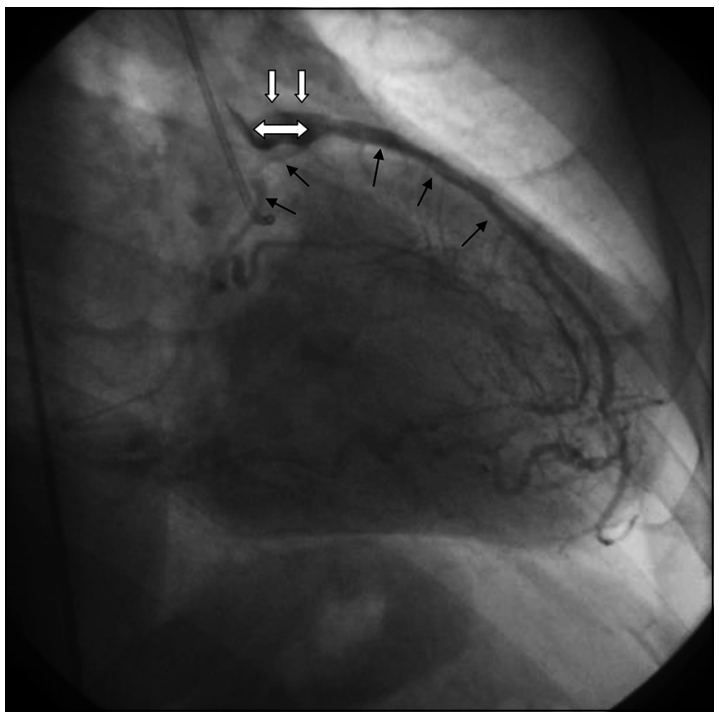

Figura 3. Llene completo de $\mathrm{ACl}(\longrightarrow)$ hasta el $\mathrm{TCl}($ (\) que drena en arteria pulmonar $(\Longleftrightarrow)$.

terior y oclusión del ostium de ACI en el tronco de la AP. Después de 5 días de óptima recuperación, fue dada de alta. A los 21 días post cirugía la paciente refirió mejoría en su capacidad funcional y ausencia de angor o disnea. A los 2 años de la cirugía la paciente permanecía asintomática. A los 5 años permanecía sin nuevas hospitalizaciones. 


\section{Discusión}

Angelini, para diferenciar una variante anatómica de una arteria coronaria de una anomalía, define una anomalía coronaria como la presencia de cualquier alteración coronaria que ocurra en menos del $1 \%$ de la población ${ }^{4}$. Este es el caso del síndrome de ALCAPA. Esta es una anomalía del origen del ostium de la ACI cuya incidencia se aproxima a $1 / 300.00$ nacidos vivos y representa entre 0,26 y $0,46 \%$ de las cardiopatías congénitas ${ }^{1,5}$. En adultos tiene una incidencia no definida a nivel poblacional, en estudios angiográficos se describe una incidencia de $0,008 \%{ }^{6}$ (datos mostrados en el mayor registro angiográfico publicado hasta ahora, con más de 120.000 pacientes). En Chile, hay un solo estudio publicado en referencia a anomalías coronarias, con 10.000 casos y no describe ningún caso de ALCAPA ${ }^{7}$, similar a lo descrito en España, en 13.500 pacientes, sin ningún caso ${ }^{8}$.

La gran mayoría de las anomalías coronarias no tienen significancia clínica. Sin embargo, en este síndrome el pronóstico sin cirugía es desalentador, pues entre 85 y $90 \%$ de los pacientes muere dentro del primer año de vida ${ }^{2}$. Esta presentación clínica catastrófica se denomina forma infantil o síndrome de Bland-White-Garland, determinado por la intensa isquemia miocárdica que se produce al disminuir la perfusión coronaria izquierda desde AP, debido a la baja de presión y oxigenación de la AP que ocurre fisiológicamente durante la infancia temprana, o cese del patrón fetal. La forma adulta solo es posible en aquellos casos en que hay un gran desarrollo de colaterales desde el sistema coronario derecho hacia el izquierdo, estableciéndose un flujo retrógrado desde la ACI hacia la $\mathrm{AP}^{9}$. No obstante, el número y calibre excesivo de colaterales producen un abundante cortocircuito arteriovenoso desde la ACD hacia la AP, dando origen a un síndrome de robo coronario, pudiendo así producir sintomatología en el adulto ${ }^{10}$.

En una revisión por Yau et al de 151 casos adultos, se describe la presentación clínica y los hallazgos de laboratorio en estos pacientes ${ }^{3}$. Existe una predominancia de sexo femenino del orden de 2:1. La mayoría (68\%) presentaba síntomas subagudos, tales como angina de pecho, palpitaciones o fatiga. El 18\% presenta condiciones amenazantes para la vida: arritmias ventriculares, síncope o muerte súbita, cuya edad promedio de aparición es de $33 \pm 14$ años. El resto era asintomático. Nuestra paciente presentó angina de esfuerzo como síntoma inicial. Al examen físico es frecuente el hallazgo de un soplo sistólico de regurgitación mitral atribuido a la disfunción de músculos papilares producto de la isquemia. Otro soplo, menos frecuente, corresponde a un soplo continuo similar al ductus persistente, causado por el exceso de circulación colateral. En $96 \%$ de los electrocardiogramas (ECG) existen evidencias de isquemia o infarto previo anterolateral, excepcionalmente el ECG es normal, como ocurrió en nuestro caso. El 46\% de los ecocardiogramas Doppler son sugerentes del diagnóstico al mostrar el flujo de las colaterales, la ACD sobredesarrollada o el flujo retrógrado de la ACI. El test de esfuerzo y el cintigrama miocárdico detectan isquemia en 85 y $87 \%$ de los exámenes, respectivamente. En el caso descrito el ecocardiograma no mostró alteraciones significativas y el test de esfuerzo fue claramente sugerente de isquemia.

El patrón de oro para el diagnóstico de ALCAPA es la coronariografía. Se requiere de tres criterios para su certeza: llene retrógrado de la ACI, el nacimiento de ésta desde el tronco de la AP y la ausencia de origen aórtico de la $\mathrm{ACI}^{11}$. Los tres criterios los cumplió nuestra paciente (ver fotos). Como alternativa, el diagnóstico puede certificarse mediante la visualización directa del origen de la ACI desde la AP mediante otras técnicas de imagen como la tomografía computarizada o la resonancia nuclear magnética. Esta última ha adquirido ventaja, ya que permite conocer la existencia de isquemia subendocárdica crónica, evaluar la función mitral y visualizar otros defectos congénitos asociados ${ }^{12}$.

El tratamiento definitivo de esta anomalía es la cirugía. Las primeras alternativas quirúrgicas datan de 1959 y consistían en la creación de un sistema coronario único que dependiera solamente de la ACD, con el objetivo de revertir el flujo retrogrado de la ACI y así evitar el fenómeno de robo coronario ${ }^{3}$. Entre estas técnicas de ha descrito el ligamiento del origen de la ACI, e incluso existen casos de cierre percutáneo ${ }^{13}$. Esta propuesta fue posteriormente abandonada por su asociación con alta mortalidad precoz y a largo plazo y a la falta de mejoría de la función ventricular en el seguimiento ${ }^{14}$. De estos ensayos se deduce la necesidad de un sistema de doble riego coronario, que se asemeje a lo natural, el cual ha demostrado mejorar los síntomas, la función ventricular 
izquierda, la severidad de la insuficiencia mitral y la mortalidad ${ }^{16}$. La técnica quirúrgica que permite volver la anatomía a lo ideal es la reimplantación de la ACI en la aorta ${ }^{15}$. Sin embargo, en adultos esta técnica no es usualmente utilizada debido a que la rigidez de los tejidos no permitiría una reimplantación de esa distancia ${ }^{16}$. La técnica Takeuchi, un túnel intrapulmonar desde la aorta, reestablece el flujo arterial en la ACI, pero tiene mayor riesgo de complicaciones ${ }^{17}$. Actualmente se considera que aquellos adultos en los cuales no es posible la reimplantación de la ACI en la aorta, la cirugía de elección es el bypass coronario arterial por injerto y ligamiento del origen de la ACI, procedimiento que parece tener una baja morbimortalidad ${ }^{18}$. Esto se decidió en el caso descrito, con buen resultado inicial y a largo plazo. En aquellos pacientes cuya función cardiaca no mejora a pesar de la cirugía, puede plantearse el trasplante cardiaco ${ }^{19}$.

Respecto a la insuficiencia mitral asociada, si existe y es significativa, se recomienda observar su evolución post restablecimiento del flujo coronario izquierdo, debido a que la mayoría de los pacientes mejorarán su función mitral ${ }^{20}$. Producto de los fenómenos de remodelación ventricular y presencia de cicatrices, independiente si recibieron cirugía o no, los pacientes no están exentos de un riesgo significativo de desarrollar arritmias ventriculares. Es recomendable evaluar evidencia de taquiarritmias malignas mediante estudio electrofisiológico y presencia de cicatrices en resonancia magnética cardiaca para plantear la instalación de un desfibrilador automático implantable ${ }^{16}$.

En el caso clínico presentado abre el espectro diagnóstico, aunque muy improbable, que un cuadro anginoso clásico, incluso con factores de riesgo cardiovascular evidentes, pueda corresponder a un fenómeno etiológico diferente de la enfermedad coronaria aterosclerótica, como lo es el síndrome de ALCAPA.

Destacamos, además, los hallazgos de laboratorio y los resultados de la cirugía de bypass coronario por injerto y ligamiento de la ACI en su nacimiento a nivel de la AP en este síndrome, no descritos previamente en nuestro país.

\section{Referencias}

1. Keith JD. The anomalous origin of the left coronary artery from the pulmonary artery. Br Heart J 1959; 21 (2): 149-61.
2. Wesselhoeft H, Fawcett JS, Johnson AL. Anomalous origin of the left coronary artery from the pulmonary trunk. Its clinical spectrum, pathology, and pathophysiology, based on a review of 140 cases with seven further cases. Circulation 1968; 38 (2): 403-25.

3. Yau JM, Singh R, Halpern EJ, Fischman D. Anomalous origin of the left coronary artery from the pulmonary artery in adults: a comprehensive review of 151 adult cases and a new diagnosis in a 53-year-old woman. Clin Cardiol 2011; 34 (4): 204-10.

4. Angelini P. Normal and anomalous coronary arteries: definitions and classification. Am Heart J 1989; 117 (2): 418-34.

5. Askenazi J, Nadas AS. Anomalous left coronary artery originating from the pulmonary artery. Report on 15 cases. Circulation 1975; 51 (6): 976-87.

6. Yamanaka O, Hobbs R. Coronary Artery Anomalies in 126, 595 patients undergoing Coronary Arteriography. Catheterization and Cardiovascular Diagnosis 1990; 21: 28-40.

7. Ugalde H, Ramírez A, Ugalde D, Farías E, Silva A. Nacimiento anómalo de las arterias coronarias en 10.000 pacientes adultos sometidos a coronariografía. Rev Med Chile 2010; 138: 7-14.

8. Barriales R, Moris C, López A, Hernández L, San Roman L, Barriales V, Testa A, De la Hera J, et al. Anomalías congénitas de las arterias coronarias del adulto descritas en 31 años de estudios coronariográficos en el Principado de Asturias: principales características angiográficas y clínicas. Rev Esp Cardiol 2001; 21: 28-40.

9. Edwards JE. Anomalous coronary arteries with special reference to arteriovenous-like communications. Circulation 1958; 17 (6): 1001-6.

10. Baue AE, Baum S, Blakemore WS, Zinsser HF. A later stage of anomalous coronary circulation with origin of the left coronary artery from the pulmonary artery. Coronary artery steal. Circulation 1967; 36 (6): 87885.

11. Culham JAG. Abnormalities of the Coronary Arteries. En: Freedom RM, Mawson JB, Yoo SJ, Benson LN, Editores, Congenital Heart Disease. Textbook of Angiocardiography. Armonk, NY: Futura Publishing Company Inc., 1997. p. 862-6.

12. Komócsi A, Simor T, Tóth L, Szabados S, Mágel F, Pintér $\mathrm{T}$, et al. Magnetic resonance studies in management of adult cases with Bland-White-Garland syndrome. Int J Cardiol 2007; 123 (1): e8-11.

13. Bimal F, Harikrishman S, Titus T, Tharakan J. Percutaneus coil closure of recanalised anomalous origin of left coronary artery from pulmonary artery. Int J Cardiol 2002; 83 (3): 281-3. 
14. Kayalr N, Burkhart H, Dearani J, Cetta F, Schaff H. Congenital coronary anomalies and surgical treatment. Congenit Heart Dis 2009; 4 (4): 239-51.

15. Lugones I, Kreutzer C, Román M, Schlichter J. Origen anómalo de la coronaria izquierda en la arteria pulmonar: resultados de la cirugía correctora. Rev Argent Cardiol 2010; 78 (5): 411-6.

16. Quah JX, Hofmeyr L, Haqqani H, Clarke A, Rahman A, Pohlner $\mathrm{P}$, et al. The management of the older adult patient with anomalous left coronary artery from the pulmonary artery syndrome: a presentation of two cases and review of the literature. Congenit Heart Dis 2014; 9 (6): E185-94.

17. Backer CL, Stout MJ, Zales VR, Muster AJ, Weigel TJ, Idriss FS, et al. Anomalous origin of the left coronary artery. A twenty-year review of surgical management. J Thorac Cardiovasc Surg 1992; 103 (6): 1049-57.

18. Rajbanshi BG, Burkhart HM, Schaff HV, Daly RC, Phillips SD, Dearani JA. Surgical strategies for anomalous origin of coronary artery from pulmonary artery in adults. J Thorac Cardiovasc Surg 2014; 148 (1): 220-4.

19. Nair KK, Zisman LS, Lader E, Dimova A, Canver CC. Heart transplant for anomalous origin of left coronary artery from pulmonary artery. Ann Thorac Surg 2003; 75 (1): 282-4.

20. Ben ali W, Metton O, Roubertie F, Pouard P, Sidi D, Raisky O, et al. Anomalous origin of the left coronary artery from the pulmonary artery: late results with special attention to the mitral valve. Eur J Cardiothorac Surg 2009; 36 (2): 244-8. 